CARPATHIAN J. MATH.

Volume 37 (2021), No. 2,

Pages 339 - 344
Online version at https : //www . carpathian. cunbm . utcluj. ro/

Print Edition: ISSN 1584 - 2851; Online Edition: ISSN 1843 - 4401

DOI: https://doi.org/10.37193/CJM.2021.02.18

Dedicated to Prof. Ioan A. Rus on the occasion of his $85^{\text {th }}$ anniversary

\title{
Ulam-Hyers stability and exponentially stable evolution equations in Banach spaces
}

\author{
ADRIANA BUICĂ
}

\begin{abstract}
We show that uniformly exponentially stable abstract linear evolution equations are Ulam-Hyers stable on $[a, \infty)$. Moreover, we prove that this property is maintained when perturbing this type of equations with a nonlinear term having a small Lipschitz constant. These results complement the literature on Ulam-Hyers stability, a special relation having with some works of I. A. Rus.
\end{abstract}

\section{INTRODUCTION}

There is a huge literature on Ulam-Hyers (UH) stability of functional equations, including, of course, both ordinary and partial differential equations. Most of the studies are made on linear equations. But some nonlinear equations which, in some sense are very close to be linear, are UH stable, too. It is also very important to distinguish whether this type of stability holds on compact intervals, or unbounded intervals. It seems that UH stability on a compact interval is a property of any linear differential system and of the most of the nonlinear ones. I. A. Rus emphasized and proved this using the Gronwall Lemmas technique and other techniques in $[5,6,7,8]$. Here we deal with abstract evolution equations in Banach spaces, both linear and nonlinear perturbations of linear ones, on unbounded intervals of the form $[a, \infty)$. In this framework are included the finite dimensional differential systems, delay differential systems, very general parabolic partial differential equations, both autonomous and nonautonomous. The main assumption on the linear evolution equation is its exponential stability. For the nonlinear perturbation we assume that it has a sufficiently small Lipschitz constant. We also use the Gronwall Lemmas technique. Moreover, our results complement Theorem 4.2 in [6]. Namely, we show that uniformly exponentially stable abstract linear evolution equations are UlamHyers stable on $[a, \infty)$. We also prove that this property is maintained when perturbing this type of equations with a nonlinear term having a small Lipschitz constant.

\section{MAIN RESULTS}

Let $(X,|\cdot|)$ be a real or complex Banach space. The zero vector in $X$ will be denoted by $0 . \mathcal{L}(X)$ will stand for the space of bounded linear operators from $X$ into itself. The corresponding norm in $\mathcal{L}(X)$ will also be denoted by $|\cdot|$. The identity operator on $X$ is $I \in \mathcal{L}(X)$. For notations and the next two notions we used $[2,3]$.

Definition 2.1. A family of operators $\{U(\theta, \tau)\}_{\theta \geq \tau} \subset \mathcal{L}(X)$, with $\theta, \tau \in \mathbb{R}$, is called an evolution family if

(i) $U(\theta, s) U(s, \tau)=U(\theta, \tau)$ and $U(\theta, \theta)=I$ for all $\theta \geq s \geq \tau$; and

Received: 25.01.2021. In revised form: 31.05.2021. Accepted: 01.06.2021

2010 Mathematics Subject Classification. 34G10, 34G20, 34D10.

Key words and phrases. Ulam-Hyers stability, unbounded interval, evolution famil, nonautonomous, mild solution, exponential stability, small Lipschitz constant. 
(ii) for each $x \in X$, the function $(\theta, \tau) \mapsto U(\theta, \tau) x$ is continuous for $\theta \geq \tau$.

Definition 2.2. An evolution family $\{U(\theta, \tau)\}_{\theta \geq \tau}$ is called uniformly exponentially stable if there exist real constants $M \geq 1$ and $\omega>0$ such that

$$
|U(\theta, \tau)| \leq M e^{-\omega(\theta-\tau)}, \quad \theta \geq \tau .
$$

In the proof of the main result we will use the following Gronwall-type Lemma. We include its proof for completeness.

Lemma 2.1. Fix $a \in \mathbb{R}$. Take the functions $u \in C([a, \infty)), \alpha \in C^{1}([a, \infty))$, and the real constant $\beta>0$. If

$$
u(t) \leq \alpha(t)+\beta \int_{a}^{t} u(s) d s, \quad t \geq a
$$

then

$$
u(t) \leq \alpha(a) e^{\beta(t-a)}+\int_{a}^{t} \alpha^{\prime}(s) e^{\beta(t-s)} d s, \quad t \geq a .
$$

Proof. Denote $v(t)=\alpha(t)+\beta \int_{a}^{t} u(s) d s$. Then

$$
u \leq v, \quad v^{\prime}=\alpha^{\prime}+\beta u \leq \alpha^{\prime}+\beta v, \quad v(a)=\alpha(a)
$$

From here we obtain $v^{\prime}-\beta v \leq \alpha^{\prime}$ on $[a, \infty)$. First we multiply this last relation with $e^{-\beta t}$, then integrate from $a$ to $t$. After some small computations, using eventually that $u \leq v$, one obtains the conclusion.

Now we state and prove the most general result of this work. This theorem concludes the Ulam-Hyers stability of mild solutions of abstract nonautonomous evolution equations, but we preferred to not give such a notion. Anyway, in subsections 2.1 and 2.2 we stated results that conclude the UH stability of finite dimensional differential systems and, respectively, of an abstract evolution equation whose linear part is the generator of a $C_{0}$-semigroup. Of course, these results are consequences of the next theorem.

Theorem 2.1. Let $\{U(\theta, \tau)\}_{\theta \geq \tau}$ be a uniformly exponentially stable evolution family on $X$. Let the constants $M \geq 1$ and $\omega>0$ be like in Definition 2.2. Fix $a \in \mathbb{R}, \varepsilon>0, L>0$ and the continuous functions $g:[a, \infty) \rightarrow X$ and $f:[a, \infty) \times X \rightarrow X$. Assume that

(i) $|g(s)| \leq \varepsilon, s \in[a, \infty)$.

(ii) $|f(s, y)-f(s, x)| \leq L|x-y|, s \in[a, \infty), x, y \in X$.

(iii) $L<\omega / M$.

Let $\eta \in X$. Then, each of the integral equations

$$
\begin{aligned}
& y(t)=U(t, a) \eta+\int_{a}^{t} U(t, s)[f(s, y(s))+g(s)] d s, \\
& x(t)=U(t, a) \eta+\int_{a}^{t} U(t, s) f(s, x(s)) d s,
\end{aligned}
$$

has a unique solution in $C([a, \infty), X)$, denoted $\varphi$ and, respectively, $\psi$. Moreover, they satisfy

$$
|\varphi(t)-\psi(t)| \leq \varepsilon \frac{M}{\omega-L M}, \quad t \in[a, \infty) .
$$


Proof. Consider $b>a$ arbitrary but fixed and the operator

$$
B: C([a, b], X) \rightarrow C([a, b], X)
$$

defined for any $x \in C([a, b], X)$ and for any $t \in[a, b]$ by

$$
B(x)(t)=U(t, a) \eta+\int_{a}^{t} U(t, s)[f(s, x(s))+g(s)] d s .
$$

It is known that $C([a, b], X)$ with the norm $\|x\|=\max _{t \in[a, b]}|x(t)|$ is a Banach space. We claim that $B$ is a contraction with the Lipschitz constant $L M / \omega$. For any $x_{1}, x_{2} \in C([a, b], X)$ and $t \in[a, b]$ we have

$$
\begin{aligned}
\left|B\left(x_{1}\right)(t)-B\left(x_{2}\right)(t)\right| & =\left|\int_{a}^{t} U(t, s)\left[f\left(s, x_{1}(s)\right)-f\left(s, x_{2}(s)\right)\right] d s\right| \\
& \leq L \int_{a}^{t}|U(t, s)| \cdot\left|x_{1}(s)-x_{2}(s)\right| d s \\
& \leq L M\left\|x_{1}-x_{2}\right\| \int_{a}^{t} e^{-\omega(t-s)} d s \\
& \leq \frac{L M}{\omega}\left\|x_{1}-x_{2}\right\| .
\end{aligned}
$$

Then

$$
\left\|B\left(x_{1}\right)-B\left(x_{2}\right)\right\| \leq \frac{L M}{\omega}\left\|x_{1}-x_{2}\right\| .
$$

Thus, the claim is proved. The Contraction Mapping Principle assures the existence of a unique fixed point of $B$ in $C([a, b], X)$. Thus, equation (2.1) has a unique continuous solution defined on the interval $[a, b]$. Since $b>a$ is arbitrary, we deduce the existence of a unique continuous solution defined on the interval $[a, \infty)$, denote it by $\varphi$. Note that equation (2.2) can be seen as a particular case of (2.1) (with $g=0$ ). Denote the solution of (2.2) by $\psi$. In the sequel we intend to estimate the distance between $\varphi$ and $\psi$. For any $t \in[a, \infty)$ we have

$$
\begin{aligned}
|\psi(t)-\varphi(t)| & =\left|\int_{a}^{t} U(t, s)[f(s, \psi(s))-f(s, \varphi(s))] d s+\int_{a}^{t} U(t, s) g(s) d s\right| \\
& \leq \int_{a}^{t}|U(t, s)| \cdot|f(s, \psi(s))-f(s, \varphi(s))| d s+\int_{a}^{t}|U(t, s)| \cdot|g(s)| d s \\
& \leq L M \int_{a}^{t} e^{-\omega(t-s)}|\psi(s)-\varphi(s)| d s+\varepsilon M \int_{a}^{t} e^{-\omega(t-s)} d s .
\end{aligned}
$$

With the following notations

$$
u(t)=|\psi(t)-\varphi(t)| e^{\omega t}, \quad \alpha(t)=\varepsilon M \int_{a}^{t} e^{\omega s} d s, \quad \beta=L M
$$

we obtain that

$$
u(t) \leq \beta \int_{a}^{t} u(s) d s+\alpha(t), \quad t \in J
$$

Now we apply Lemma 2.1, use the relations $\alpha(a)=0, \alpha^{\prime}(t)=\varepsilon M e^{\omega t}$ and obtain

$$
u(t) \leq \varepsilon M e^{L M t} \int_{a}^{t} e^{(\omega-L M) s} d s .
$$

After we multiply with $e^{-\omega t}$, compute the integral, and replace the expression of $u(t)$, we obtain (2.3). 
Remark 2.1. We consider that it is worth to point out here the connections between three notions of asymptotic stability of evolution families, see [3].

We say that an evolution family $\{U(\theta, \tau)\}_{\theta \geq \tau}$ is asymptotically stable if $\lim _{t \rightarrow \infty} U(t, \tau) x=0$ for all $x \in X$ and, respectively, it is uniformly asymptotically stable if given any $\varepsilon>0$ there exists a $T(\varepsilon)>0$ such that $|U(t, \tau)|<\varepsilon$ wherever $t>T(\varepsilon)+\tau$. It is not difficult to see that uniform exponential stability implies uniform asymptotic stability which implies asymptotic stability. It is known from [3] that uniform asymptotic stability implies uniform exponential stability. However, asymptotic stability does not in general imply uniform asymptotic stability, a property which is true if $\mathrm{X}$ is finite-dimensional.

2.1. Differential systems in $\mathbb{R}^{n}$ or $\mathbb{C}^{n}$. Let the Banach space $X$ be either $\mathbb{R}^{n}$ or $\mathbb{C}^{n}$. Let $A \in C(\mathbb{R}, \mathcal{L}(X))$. For each $a \in \mathbb{R}$ let $U(t, a)$ be the fundamental matrix solution of the system $x^{\prime}=A(t) x$ such that $U(a, a)$ is the identity matrix. Then $\{U(\theta, \tau)\}_{\theta>\tau}$ is an evolution family. In addition, we have that $U(\theta, \tau)$ is well defined for $\theta<\tau$ and we have $[U(\theta, \tau)]^{-1}=U(\tau, \theta)$ for all $\theta, \tau \in \mathbb{R}$. We say that the system $x^{\prime}=A(t) x$ is uniformly exponentially stable whenever $\{U(\theta, \tau)\}_{\theta \geq \tau}$ is uniformly exponentially stable. In the particular case when $A$ is a constant function, we have that the autonomous system $x^{\prime}=A x$ is (uniformly) exponentially stable if and only if $\Re(\lambda)<0$ for all $\lambda \in \sigma(A)$. Here $\sigma(A) \subset \mathbb{C}$ is the spectrum of the matrix $A$ and $\Re(\lambda)$ denotes the real part of $\lambda$. Moreover, a constant $\omega>0$ such that $\Re(\lambda)<-\omega$ for all $\lambda \in \sigma(A)$, can appear in Definition 2.2. All the above are known results from the classical theory of ordinary differential equations, as can be found, for example, in [1].

In addition, let $f \in C([a, \infty) \times X, X)$ and $m>0$. We say that the system

$$
x^{\prime}=A(t) x+f(t, x)
$$

is Ulam-Hyers stable on the interval $[a, \infty)$ with constant $m$ if for any $\varepsilon>0$ and for any solution $\psi \in C^{1}([a, \infty), X)$ of

$$
\left|x^{\prime}-A(t) x-f(t, x)\right| \leq \varepsilon
$$

there exists a solution $\varphi \in C^{1}([a, \infty), X)$ of system (2.4) such that

$$
|\psi(t)-\varphi(t)| \leq m \varepsilon, \quad t \in[a, \infty) .
$$

As a consequence of Theorem 2.1 we obtain the following result.

Theorem 2.2. Assume that the system $x^{\prime}=A(t) x$ is uniformly exponentially stable and let $M \geq 1$ and $\omega>0$ be like in Definition 2.2. Assume that there exists $0<L<\omega / M$ such that

$$
|f(s, y)-f(s, x)| \leq L|x-y|, \text { for all } s \in[a, \infty), \quad x, y \in X .
$$

Then system (2.4) is Ulam-Hyers stable on the interval $[a, \infty)$ with constant $m=M /(\omega-L M)$.

Corollary 2.1. A uniformly exponentially stable system in $\mathbb{R}^{n}$ or $\mathbb{C}^{n}$

$$
x^{\prime}=A(t) x
$$

is Ulam-Hyers stable on the interval $[a, \infty)$ with constant $m=M / \omega$, where $M \geq 1$ and $\omega>0$ are like in Definition 2.2. 
2.2. Semigroups. For the definition of a $C_{0}$-semigroup and other useful results we used $[4,3]$.

Definition 2.3. If the evolution family $\{U(\theta, \tau)\}_{\theta \geq \tau}$ on the Banach space $X$ satisfies in addition

$$
U(\theta, \tau) x=U(\theta-\tau, 0) x, \quad \theta \geq \tau, \quad x \in X,
$$

then it is called a $C_{0}$-semigroup.

Assume from now that $\{U(\theta, \tau)\}_{\theta \geq \tau}$ is a $C_{0}$-semigroup. An important remark is that there exists a dense set $D \subset X$ and a linear operator $A: D \rightarrow X$ such that if $x \in D$,

$$
\lim _{\theta \downarrow 0} \frac{1}{\theta} U(\theta, 0) x=A x \text {. }
$$

The mapping $A$ is in general unbounded and is called the infinitesimal generator of the semigroup. Sometimes the following notation is used

$$
e^{t A}:=U(t, 0), \quad t \geq 0
$$

and it is said that $\left\{e^{t A}\right\}_{t \geq 0}$ is a one-parameter $C_{0}$-semigroup.

Let $f \in C([a, \infty) \times X, X)$ and consider the abstract evolution equation

$$
x^{\prime}+A x=f(t, x),
$$

and the abstract evolution inequation

$$
\left|x^{\prime}+A x-f(t, x)\right| \leq \varepsilon .
$$

We say that $\varphi \in C([a, \infty), X)$ is a mild solution of equation (2.5) if $\varphi$ is a solution of the integral equation (2.2) with $\eta=\varphi(a)$.

We say that $\psi \in C([a, \infty), X)$ is a mild solution of inequation (2.6) if there exists $g \in$ $C([a, \infty))$ with $|g(s)| \leq \varepsilon, s \in[a, \infty)$ such that $\psi$ is a solution of the integral equation (2.1) with $\eta=\psi(a)$.

Let $m>0$. We say that the evolution equation (2.5) is Ulam-Hyers stable on the interval $[a, \infty)$ with constant $m$ if for any $\varepsilon>0$ and for any mild solution $\psi \in C([a, \infty), X)$ of inequation (2.6) there exists a mild solution $\varphi \in C([a, \infty), X)$ of $(2.5)$ such that

$$
|\psi(t)-\varphi(t)| \leq m \varepsilon, \quad t \in[a, \infty) .
$$

As a consequence of Theorem 2.1 we obtain the following result.

Theorem 2.3. Let $A: D \subset X \rightarrow X$ be the infinitesimal generator of a uniformly exponentially stable $C_{0}$-semigroup $\{U(\theta, \tau)\}_{\theta \geq \tau}$. Let $M$ and $\omega$ be like in Definition 2.2. Assume that there exists $0<L<\omega / M$ such that

$$
|f(s, y)-f(s, x)| \leq L|x-y|, \text { for all } s \in[a, \infty), \quad x, y \in X .
$$

Then the abstract evolution equation (2.5) is Ulam-Hyers stable on the interval $[a, \infty)$ with constant $m=M /(\omega-L M)$.

Corollary 2.2. Let $A: D \subset X \rightarrow X$ be the infinitesimal generator of a uniformly exponentially stable $C_{0}$-semigroup $\{U(\theta, \tau)\}_{\theta \geq \tau}$. Let $M$ and $\omega$ be like in Definition 2.2. Then the abstract evolution equation

$$
x^{\prime}=A x
$$

is Ulam-Hyers stable on the interval $[a, \infty)$ with constant $m=M / \omega$.

Acknowledgements. This work was partially supported by a grant of the Romanian Ministry of Education and Research, CNCS - UEFISCDI, project number PN-III-P1-1.1-TE2019-1306, within PNCDI III.

The author thanks Professor Rus for useful discussions during the preparation of this work. 


\section{REFERENCES}

[1] Chicone, C., Ordinary Differential Equations with applications, Springer, 2006

[2] Chicone, C. and Latushkin, Y.,Evolution Semigroups in Dynamical Systems and Differential Equations, American Mathematical Society, 1999

[3] Datko, R., Uniform asymptotic stability of evolutionary processes in a Banach space, SIAM J. Math. Anal., 3 (1972), $428-445$

[4] Engel, Klaus-Jochen and Nagel, R., One-parameter Semigroups for Linear Evolution Equations, Springer 2000

[5] Rus, I. A., Gronwall lemma approach to the Hyers-Ulam-Rassias stability of an integral equation Nonlinear analysis and variational problems, 147-152, Springer Optim. Appl., 35, Springer, New York, 2010

[6] Rus, I. A., Ulam stabilities of ordinary differential equations in a Banach space, Carpathian J. Math., 26 (2010), No. $1,103-107$

[7] Rus, I. A., Ulam stabilities of ordinary differential equations, Studia Univ. Babeş-Bolyai Math., 54 (2009), No. 4, $125-133$

[8] Rus, I. A., Remarks on Ulam stability of the operatorial equations, Fixed Point Theory, 10 (2009), No. 2, 305-320

Departamentul DE MATEMATiCĂ

UNIVERSITATEA BABEŞ-BOLYAI

STR. KOGĂLNiCEANU 1, 400084 ClUJ-NAPOCA, ROMANIA

E-mail address: abuica@math.ubbcluj.ro 